of our map is a maximal ideal and we see that Statements S2 and S3 are violated. This concludes the proof of our theorem.

\title{
REFERENCE
}

1. O. Zariski, A new proof of Hilbert's Nullstellensatz, Bull. Amer. Math. Soc. vol. 53 (1947) p. 362.

Princeton University

\section{ON ORDERED SKEW FIELDS}

T. SZELE

In this paper we shall give a necessary and sufficient condition that a skew field can be ordered; moreover, that the ordering of an ordered skew field $K$ can be extended to an ordering of $L, L$ being a given extension of $K$. The first of these two results generalizes to skew fields a theorem of E. Artin and O. Schreier [1],1 according to which a commutative field can be ordered if and only if it is formally real. The second result generalizes in the same sense a recent theorem of J. P. Serre [2].

Our considerations are based on the following definition.

Definition. A skew field is said to be ordered if in its multiplicative group a subgroup of index 2 is marked out which is also closed under addition.

Hence a skew field can be ordered if and only if its multiplicative group has a subgroup of index 2 which is also closed under addition.

We shall now prove the following theorem.

THEOREM 1. A skew field $K$ can be ordered if and only if -1 cannot be represented as a sum of elements of the form

$$
\underset{a_{1} a_{2}^{2}}{2} \ldots a_{k}^{2} \quad\left(a_{i} \in K, i=1,2, \cdots, k\right) .
$$

REMARK. This property can be considered as a generalization of the notion "formally real" to the case of skew fields.

The necessity of the condition in Theorem 1 is obvious. In order to prove its sufficiency we consider a skew field $K$ in which -1 cannot be represented as a sum of elements (1). We shall show that the

Received by the editors September 20, 1951.

1 Numbers in brackets refer to the bibliography at the end of the paper. 
multiplicative group $K^{*}$ of $K$ has a subgroup of index 2 which is also closed under addition.

Let $S$ be the subset of all (finite) sums of elements (1) in $K$ with every $a_{i} \neq 0$. Clearly $0 \notin S$, for in the contrary case we should have a relation

$$
-a_{1}^{2} a_{2}^{2} \cdots a_{k}^{2}=b_{1}^{2} b_{2}^{2} \cdots b_{l}^{2}+\cdots
$$

from which would follow, by multiplication on the right by $a_{k}^{-2}, \cdots$, $a_{1}^{-2}$, that $-1 \in S$ in contradiction to our hypothesis. On the other hand, one can see immediately that

$$
\begin{aligned}
& s \in S, s^{\prime} \in S \text { imply } s s^{\prime} \in S \text { and } s+s^{\prime} \in S, \\
& s \in S \text { implies } s^{-1}=s \cdot s^{-2} \in S, \\
& s \in S, z \in K^{*} \text { imply } z^{-1} s z \in S .
\end{aligned}
$$

Hence $S$ is a proper invariant subgroup of $K^{*}$ which is closed under addition. The order of each element $(\neq 1)$ in $K^{*} / S$ being $2, K^{*} / S$ is abelian. Consequently any subgroup $P$ of $K^{*}$ which contains $S$ is invariant in $K^{*}$.

Now we define $P$ as a maximal subgroup of $K^{*}$ for which

$$
S \subseteq P,-1 \notin P \text {, and } P \text { is closed under addition. }
$$

The existence of such a group $P$ follows immediately from Zorn's lemma. We have only to show that the decomposition

$$
K^{*}=P \cup(-1) P
$$

holds. Suppose (3) is not true. Then there exists an element $d$ such that

$$
d \in K^{*}, \quad d \notin P, \quad-d \notin P .
$$

Consider the set $P^{\prime}$ of all elements

$$
u+v d \quad(u, v \in\{P, 0\} \text { but not } u=v=0) .
$$

Then, by (4), $P^{\prime}$ contains $P$ as a proper subset. On the other hand we shall show that $P^{\prime}$ is a subgroup of $K^{*}$ having the properties (2) (with $P^{\prime}$ instead of $P$ ). This is a contradiction to the maximal property of $P$, which will complete the proof.

First we show that $0 \notin P^{\prime}$. Indeed, by the exclusion of $u=v=0$, $u+v d=0$ would imply that $v \neq 0$ and hence that $-d=v^{-1} u \in P$, in contradiction to (4). Moreover, if $u_{1}+v_{1} d$ and $u_{2}+v_{2} d$ are arbitrary elements of $P^{\prime}$, we have 
(5) $\quad\left(u_{1}+v_{1} d\right)\left(u_{2}+v_{2} d\right)=\left(u_{1} u_{2}+v_{1} d v_{2} d\right)+\left(u_{1} v_{2} d+v_{1} d u_{2}\right)$.

But since $P$ is an invariant subgroup of $K^{*}, d v_{2}=v_{2}^{\prime} d, d u_{2}=u_{2}^{\prime} d$ hold with suitable elements $u_{2}^{\prime}, v_{2}^{\prime} \in P$, so that (5) is an element of $P^{\prime}$. If $u+v d \in P^{\prime}$, we obtain

$$
(u+v d)^{-1}=(u+v d)(u+v d)^{-2} \in P^{\prime} .
$$

Hence $P^{\prime}$ is a group which is obviously closed under addition. Finally, $-1 \notin P^{\prime}$ for $u+v d=-1$ would imply (on account of $v \neq 0$ ) that $-d=v^{-1}(u+1) \in P$. This completes the proof.

In an analogous manner we prove the following theorem.

TheOREM 2. Let $L$ be an extension of the ordered skew field $K$. The ordering of $K$ can be extended to an ordering of $L$ if and only if -1 cannot be represented as a sum of elements

$$
p_{1} u_{1}^{2} \cdots p_{k} u_{k}^{2} \quad\left(p_{i} \in K, p_{i}>0, u_{i} \in L, i=1,2, \cdots, k\right) .
$$

REMARK. Theorem 1 is the special case of Theorem 2 in which $K$ is the prime field of characteristic zero. However, this special case seemed of sufficient interest to warrant an independent proof. Only a few remarks are now necessary to prove Theorem 2 since the proof follows the same general pattern as that of Theorem 1.

The necessity of the condition in Theorem 2 is obvious. In order to prove its sufficiency we define the subset $U$ of $L$ as the set of all (finite) sums of elements (6) with every $u_{i} \neq 0$. One can show as above that $U$ is a subgroup of the multiplicative group $L^{*}$ of $L$. That, e.g., $0 \notin U$ follows from the fact that a relation

$$
-p_{1} u_{1}^{2} \cdots p_{k} u_{k}^{2}=p_{1}^{\prime} v_{1}^{2} \cdots p_{l}^{\prime} v_{l}^{2}+\cdots
$$

would imply that

$$
-1=p_{1}^{\prime} v_{1}^{2} \cdots p_{l}^{\prime} v_{l}^{2}\left(1 \cdot u_{k}^{-2}\right)\left(p_{k}^{-1} u_{k-1}^{-2}\right) \cdots\left(p_{1}^{-1} \cdot 1^{2}\right)+\cdots,
$$

which is impossible.

Moreover $U$ is an invariant subgroup of $L^{*}$. This follows from the fact that

$$
p \in K, \quad p>0, \quad z \in L^{*}
$$

imply that

$$
z^{-1} p z=z^{-2} z p z p p^{-1}=p_{1}^{\prime} v_{1}^{2} p_{2}^{\prime} v_{2}^{2} p_{3}^{\prime}
$$

with $p_{1}^{\prime}=1, v_{1}=z^{-1}, p_{2}^{\prime}=1, v_{2}=z p, p_{3}^{\prime}=p^{-1}$. 
From the fact that each element $(\neq 1)$ of $L^{*} / U$ is of order 2, we infer as above that any subgroup $Q$ of $L^{*}$ containing $U$ is invariant in $L^{*}$.

Now we define $Q$ as a maximal subgroup of $L^{*}$ for which $U \subseteq Q$, $-1 \notin Q$, and $Q$ is closed under addition. Then one can show as above that $Q$ is a subgroup of index 2 of $L^{*}$. Since all positive elements of $K$ are contained in $U$ and consequently in $Q$, the theorem is proved.

REMARK (added April 28, 1952). I have noticed that for the case of a countable skew field $K$, Theorem 1 occurs in the book of G. Pickert, Einfiuhrung in die höhere Algebra (Göttingen, 1951), p. 238, Aufgabe 13.

\section{BIBLIOGRAPHY}

1. E. Artin and O. Schreier, Algebraische Konstruktion reeller Körper, Abh. Math. Sem. Hamburgischen Univ. vol. 5 (1926) pp. 83-115.

2. J. P. Serre, Extensions de corps ordonnes, C. R. Acad. Sci. Paris vol. 229 (1949) pp. 576-577.

Debrecen University, Hungary 